\title{
Modélisation de la régulation de la stoechiométrie des fermentations ruminales par la disponibilité en ATP et en NADH2
}

\author{
D Sauvant 1, J Van Milgen 2, P Lescoat 1 \\ Laboratoire INRA, INAPG, Institut National Agronomique Paris-Grignon, 75231 Paris Cedex 05 ; \\ 2INRA, SRNH ef SRP, St-Gilles, 35590 L'Hermitage, France
}

La plupart des modèles mécanistes du rumen publiés (Baldwin et al, 1987, J Dairy Res, 54, 107-131; Danfaer, 1990, Thesis, Report 671, NIAS, Foulum, Denmark ; Dijkstra et al, 1992 , J Nutr, 122, 2239-2256) supposent que la répartition des flux de carbone (C) entre les voies fermentaires est fixée sur la base des équations stoechiométriques de Murphy et al (1982, J Anim Sci, 55, 411-421). Cependant, de nombreuses expériences ont montré que cette répartition était variable en fonction vraisemblablement de mécanismes de régulations cellulaires. Un modèle mécaniste dynamique, de pas de temps d'intégration d'1 min, a été établi sur le principe que la disponibilité en énergie des cellules microbiennes, évaluée par le rapport ATP/ADP, modifiait, de façon rétroactive, la répartition de $C$ entre les voies fermentaires de manière à maintenir un rapport ATP/ADP dans une fourchette de variation limitée. Le même principe a été appliqué pour la disponibilité en hydrogène évaluée par le rapport NADH2/NAD. Les flux d'échanges entre les compartiments ATP et ADP, d'une part, et NADH2 et NAD, d'autre part, ont été calculés sur la base des rendements des différentes voies biochimiques cellulaires connues. Ainsi les productions molaires d'ATP et de $\mathrm{NADH} 2$ par $\mathrm{C}$ terminal, considérées sans tenir compte de la méthanogénèse, sont différentes entre l'acétate $(1,0$ et 0,95$)$ le butyrate $(0,72 ; 0,25)$ le propionate $(0,66$; $-0,3)$ et les acides gras mineurs $(0,12 ; 0,0)$. Les variations des flux de $C$ ( $F C i$ impliqués dans les différentes voies fermentaires sont supposées être des fonctions des disponibilités en énergie et hydrogène selon des lois de Michaelis : $\mathrm{FCi}=\mathrm{FCimax}(1+\mathrm{k} 1$ $\left.(\text { ATP/ADP })^{\mathrm{h} 1}+\mathrm{k} 2(\mathrm{NADH} 2 / \mathrm{NAD})^{\mathrm{h} 2}\right)^{-1}$. Les valeurs de FCimax sont fixées de manière à ce que les proportions molaires des 4 acides cités soient respectivement de $66,8,22$ et $4 \%$. Les valeurs des quatre autres paramètres permettent d'appliquer les principes de régulation évoqués ci-dessus. Ainsi une carence énergétique favorise la voie de l'acétate tandis qu'un excès d'énergie active les voies de synthèse des AGV plus longs. Cependant, le principal problème rencontré concerne la détermination des valeurs de ces paramètres qui a été faite par approximations successives compte tenu du manque de données expérimentales.

Le flux de $\mathrm{CO}_{2}$ correspond au tiers des flux de $\mathrm{C}$ impliqués pour la synthèse d'acétate, de butyrate et des acides mineurs. Le flux de $\mathrm{CH}_{4}$ dépend du flux de $\mathrm{CO}_{2}$ mais également d'un indice de développement des bactéries méthanogènes et du rapport NADH2/NAD du rumen.

Ces différents principes sont intégrés dans un modèle plus vaste (42 compartiments) de la digestion ruminale. Malgré les difficultés rencontrées pour déterminer les valeurs des paramètres, le modèle fournit des résultats réalistes de l'influence du régime sur le profil des AGV et sur l'évolution post prandiale de ce profil et celle des gaz du réticulo-rumen. 